Reverend Mr Farish", whose curiosity led to Franklin's experiment being recorded in the Philosophical Transactions. James Farish is the fall guy of Tanford's book, simply because he failed to draw the necessary conclusions from Franklin's experiment. He is "unimportant", "foolish", "naive" and perpetrator of "the Farish syndrome: failure to learn". Yet "poor Mr Farish" was a very learned man, something of a polymath, whose chief fault was reported as a reluctance to go into print. He married into the famous Gilpin family and appears in the Memoirs of Richard Gilpin. His son William was Professor of Chemistry at Cambridge. He was not a good choice as Simplicio.

Enough! Tanford has written a fine account of an intriguing aspect of chemical and biochemical history, one from which even historians can learn a great deal.

Colin Russell is in the Department of History of Science and Technology, Open University, Walton Hall, Milton Keynes MK7 6AA, UK.

\section{Universal theory}

Iwan Williams

The Formation and Evolution of Planetary Systems. Edited by H. A. Weaver and L. Danly. Cambridge University Press: 1989. Pp.353. £30, \$44.50.

The Origin of the Solar System: The Capture Theory. By John R. Dormand and Michael M. Woolfson. Ellis Horwood: 1989. Pp.230. £29.95. Distributed by John Wiley in the United States, $\$ 58.60$.

THE origin of the Earth and other planets of the Solar System has been a topic of discussion among intelligent human beings ever since they became intelligent. After Copernicus had established the true dynamical nature of the system, theories became more scientific and today we are essentially still in debate over the correctness of ideas formulated 200 years ago.

We have made some progress, of course, and we have very much more data available now, as a consequence of both space exploration and improved ground-based observation. Indeed, in the $1960 \mathrm{~s}$ and 1970 s there was an explosion in the number of workers in the field, and at one stage it was a standard joke that $\mathrm{Al}$ Cameron would produce a new theory for every meeting. The 1980 s have been much more a time for consolidation. There have been relatively few books published on the topic over the past few years, so it comes as a surprise to find two of them appearing almost simultaneously.

The Formation and Evolution of Planetary Systems is the report of a meeting on the topic and more or less presents the current consensus view. It is an edited work, with various contributors looking at different aspects of the problem, and is not therefore a comprehensive account of the formation of planets. By contrast, The Origin of the Solar System by Dormand and Woolfson presents the authors' own, more controversial, view; here we do have the (or rather a) complete story starting with interstellar material and finishing with a planetary system. As might be expected, the books are almost mutually exclusive, with no reference to Dormand and Woolfson's work in the Weaver and
Danly volume, while only the work of Cameron, of all the contributors in that book, is mentioned by Dormand and Woolfson.

It is almost self-evident, and has been so for several centuries, that there are fundamentally only two ways of making planets - either you break up something

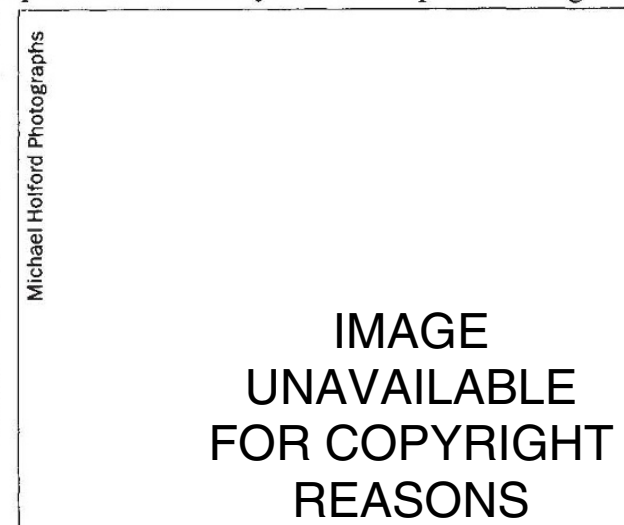

big or you build them up from small bits. Dormand and Woolfson go along the first route and envisage the capture of material from a passing proto-star by the young Sun. This material fragments under its own gravity to form spherical proto-planets on orbits not dissimilar to those of the present planets. These proto-planets would initially be gaseous but self-gravity causes any solid grains to settle to the centre. Those nearest to the Sun lose most of their outer gaseous layers while the others retain most of the gas. The book gives a very readable account of all the computer modelling that has been carried out to verify the model, which has fewer flaws than is claimed by opponents who have not studied it.

Most planetary scientists have gone along the alternative road, however, and primarily consider formation of planets through a build up of small objects, mainly by the aggregation of condensed grains within a gaseous envelope or disk surrounding the Sun. Close to the Sun the grains are composed of the non-volatile materials only, and the final aggregated planets are similar in composition, and small, as such material is not abundant. Further out water exists as ice, which results in much more material condensing and bigger embryo planets. Such embryos are able also to capture the free gas and so the end-product is a large gaseous planet.

Once a theory becomes fashionable, many people start to work on it and it then becomes possible to start an investigation at an arbitrary point in the story and possibly not continue to the final emergence of a finished planet. This is the situation with the Weaver and Danly volume, where individual chapters deal with isolated bits of the overall problem. This is fine for current workers in the field, but will not be
Model behaviour - the relative motions of the planets within the Solar System are shown on an orrery by means of clockwork mechanics. Orrerys became fashionable instruments to own during the eighteenth century when they were of practical and ornamental use. These clockwork Solar Systems were named after Charles Boyle, Earl of Orrery, for whom one was made in 1713.

This particular miniature was made by Troughton in the early part of the eighteenth century, and is at the Science Museum, London.

particularly helpful for astronomers who are specialists in other areas (of course, it was never intended that the volume should have that purpose).

These two books are very different and have different aims. The conference proceedings take a look at the current state of research as perceived by conventional wisdom and highlights potential problems which should be tackled, by both theorists and observers. Within this context, it will be useful to new workers in the field and to the older generation who have perhaps drifted into other areas. Dormand and Woolfson present a single unique theory for the formation of planets. Their book deserves to be read by the planetary science community; although I have my doubts, they may just be correct.

Iwan Williams is in the School of Mathematical Sciences, Queen Mary College, Mile End Road, London E1 4NS, UK. 